\title{
The Effect of Stretching Hamstring, Gastrocnemius, Iliopsoas and Back Muscles on Pain and Functional Activities in Patients with Chronic Low Back Pain: A Randomized Clinical Trial
}

\author{
Hamada E. Seif', Aqeel Alenazi², Sahar Mahmoud Hassan1, Shaji John Kachanathu', \\ Ashraf R. Hafez ${ }^{*}$ \\ ${ }^{1}$ Cairo UniversityHospital, Cairo University, Cairo, Egypt \\ ${ }^{2}$ Physical Therapy and Rehabilitation Department, College of Applied Medical Sciences, Salman Bin Abdulaziz \\ University, Alkharj, Saudi Arabia \\ ${ }^{3}$ Collage of Applied Medical Sciences, King Saud University, Riyadh, Saudi Arabia \\ Email: *ashrafrh1989@yahoo.com
}

Received 15 September 2015; accepted 6 November 2015; published 9 November 2015

Copyright (C) 2015 by authors and Scientific Research Publishing Inc.

This work is licensed under the Creative Commons Attribution International License (CC BY). http://creativecommons.org/licenses/by/4.0/

(c) () Op Och Access

\section{Abstract}

A back pain lasting more than 12 weeks has been defined as a chronic low back pain (LBP) [1]. More than half of people suffer from LBP [1]. The purpose of this study was to examine the effect of gastrocnemius muscle stretching in the treatment of chronic low back pain. Methods: Forty patients with chronic low back pain, ages ranging from 25 to 40 years, were recruited and divided randomly into two groups. The control group followed a physical therapy program that included stretching exercises for back, hamstring and iliopsoas muscles. Strengthening exercises for abdominal muscle and postural instructions for activities of daily living were also performed. The experimental group followed the same control-group exercises with the addition of stretching exercises for gastrocnemius muscles. The interventions for both groups consisted of three sessions per week for six weeks, each session lasting for an hour. The outcome measures were visual analog scale (VAS) for pain severity, modified fingertip to floor (FTF) for range of motion (ROM) of trunk flexion, Oswestry Disability Index (ODI), and loading X-ray to detect lumbosacral angle. Results: There was no significant difference between groups at baseline characteristics. At final follow up, the two groups were significantly different in all outcome measures in terms of pain $(p<0.01)$, modified FTF $(p<0.01)$, disability questionnaire $(p<0.01)$, and lumbosacral angle $(p<0.01)$. Con-

${ }^{*}$ Corresponding author.

How to cite this paper: Seif, H.E., et al. (2015) The Effect of Stretching Hamstring, Gastrocnemius, Iliopsoas and Back Muscles on Pain and Functional Activities in Patients with Chronic Low Back Pain: A Randomized Clinical Trial. Open Journal of Therapy and Rehabilitation, 3, 139-145. http://dx.doi.org/10.4236/ojtr.2015.34019 
clusion: Gastrocnemius manual stretching exercise is more beneficial in reducing pain and improving functions for patients with chronic low back pain when adding to stretching exercises for hamstring, iliopsoas, and back muscles, and strengthening exercise for abdominal muscles.

\author{
Keywords \\ Mechanical Back Pain, Back Pain Syndromes, Hamstring Muscle, Hip Motion, Lumbar Motion, \\ Posture, Gastrocnemius Muscle
}

\title{
1. Introduction
}

Chronic low back pain (LBP) is defined as back pain lasting more than 12 weeks, and it affects more than 50\% of the general population [1]. Over 70\% of adults have at least one episode of low back pain during their lifetimes [2]. The prevalence of LBP is higher in the young, and one important risk factor of low back pain is weakness of the superficial trunk and abdominal muscles [3]. Consequently, strengthening of these muscles is often associated with significant improvements in LBP because of its high correlation with the stability of the lumbar region [3]. The lumbar-muscle activity of patients with low back pain is low compared to healthy subjects [4]. So, conservative therapy is used in the treatment of more than $90 \%$ of patients with low back pain. For example, postural exercises have been used in the treatment for prevention of lumbar hyperlordosis [5] [6].

Mechanical low back pain recovers in six weeks to one year [7]. Low back pain is accompanied by tension and stiffness in the lower back region between the lower rib cage (T12) and the iliac crest or inferior gluteal folds and might extend to the upper legs [7]. Typical physical findings in non-specific LBP include restricted spine ROM, tight hamstring muscles, paravertebral muscle spasms, trigger points, tenderness and aggravation of symptoms on flexion or extension and straight leg raise tests [8].

The gastrocnemius muscle is a two-joint muscle that spans the knee and the ankle joints. In spite of the fact that the gastrocnemius has no relationship with the hip, this muscle can alter the Sit-and-Reach Test (SRT) results because it crosses the knee. Thus, when the patientperforms forward flexion of the trunk in a sitting position, with full extension of the knees and dorsiflexion of the ankle, all posterior muscles (hamstrings and gastrocnemius) are in tension [9]. During the standard SRT, the subjects put the ankle in dorsiflexion (DF) position with the knee fully extended. When the subject performs trunk forward flexion with the knees extended and ankles in dorsiflexion and these muscles are shortened, anuncomfortable feeling of tension in the gastrocnemius is produced. This is a limiting factor to continuing the test [10]. Hamstring muscle shortening or tightness is one of the most common findings in patients with LBP [11]. It is thought that, due to the attachment of hamstring muscles to the ischial tuberosity, the tightness of the hamstring generates posterior pelvic tilt and stop the hips from flexing during forward bending so reducing the lordosis of the lumbar spine, which can result in LBP [12] [14].

One of the most common actions in daily living is forward bending [13]. Pelvic tilt and bending alteration in the sequencing of hip and lumbar spine movement patterns during forward bending has been proposed as a risk factor for the development of LBP [15]. Changes in lumbar range of motion and motion velocity have been recognized in individuals with LBP [16]. Also, reduced hip mobility during forward bending has been reported in the literature [17]. LBP subjects demonstrate a decreased magnitude of hip flexion, but not other hip motions implying that an alteration in activity level of the hamstrings may be present in symptomatic subjects [18]. The gastrocnemius shares in knee flexion, and its shortening will indirectly affect the hamstring muscles and may limit forward bending and cause back pain due to their influence on lumbar pelvic rhythm. For this reason, stretching of the gastrocnemius should be included in the same exercise as stretching the back and hamstrings [19] [20].

Strengthening exercise for muscles is known to improve the recovery of normal joint functions after injuries [21] [22]. To reach the optimal rehabilitation effects, the strengthening exercise protocol should be adapted to variable muscle force, corresponding to changing muscle fiber length and contraction velocity [22]. We posited that the same program might be equally effective for non-injury low back pain. Therefore, the aim of this study was to compare the clinical outcomes between two different exercise programs for the management of patients with chronic low back pain. We hypothesized that individuals treated by a program which includes stretching 
exercises for back, hamstring, iliopsoas, and gastrocnemius muscles, followed by strengthening exercises for abdominal muscle and ADL postural instructions would achieve better outcomes than programs which include only stretching exercises for back, hamstring and iliopsoas without gastrocnemius muscle stretching followed by strengthening exercises for abdominal muscle and activities of daily living postural instructions designed to improve functional activities.

\section{Methods}

Subjects: Forty participants were recruited for this study from Cairo University Hospitals, with ages ranging from 25 to 40 years old, each having chronic low back pain. Patients were informed about the treatment program after random assignment into two groups. Each group included 20 patients. They were treated in the outpatient physical therapy clinic at Cairo University Hospitals. The inclusion criteria were the patient's diagnosis of low back pain, with complaint of pain for more than three months, with no pathological involvement such as disc herniation, cauda equine, tumor, spinal stenos is, or vertebral or lower extremity fracture. Each intervention consisted of three sessions per week for six weeks. The Ethical Review Board of Cairo University Hospital approved the study, and all participants singed informed consent-forms. The trial was registered with the Australian New Zealand Clinical Trials Registry with the following number: ACTRN12614000534684.

Outcome measures: All outcome measures were assessed before and immediately after the interventions at the end of week 6. A visual analog scale (VAS) was used in our study for measuring pain severity [23] [24]. It ranges from zero (no pain) to ten (unbearable pain) grades of intensity of pain. The subjects were asked to indicate the level of pain by placing a dash at the appropriate level on a $10 \mathrm{~cm}$ horizontal line. The subjects were provided with Arabic version of the VAS with "no pain" listed on the right side and "worst pain" on the left side.

A modified fingertip-to-floor test was used for measuring the range of motion of lumbar flexion. The distance between the tip of the middle finger and the floor is usually measured with a tape measure to measure changes in lumbar flexion of patients with LBP. Also, a stool with a height of $19 \mathrm{~cm}$ has been used for subjects to stand on in order to measure the ROM. The measurements were taken with the modified fingertip-to- floor method by having the patient forward bend while standing on a stool in an attempt to touch the floor with the fingertips and then measuring the distance between the tip of the middle finger and the top of the stool [25].

The Oswestry low-back pain disability index (ODI) is a low back pain disability questionnaire. It is known as the gold standard of functional outcomes of the lower back. It consists of ten sections; each section has six possible answers. Statement 1 is graded as 0 points; statement 6 is graded as 5 points. A total score of 50 is possible and would indicate 100\% disability [26] [27]. A panel of institutional experts at Cairo University Hospitals has translated and validated Oswestery Disability Index into Arabic language.

Lateral view loading $\mathrm{x}$-ray from standing position (Siemens Polyphose 50 apparatus) was used to detect any change in lordotic curve by measuring lumbo-sacral (LS) angle. It measures the meeting of two lines: a horizontal line from the superior surface of the first sacral vertebra and a horizontal line from the superior surface of the first lumbar vertebra [28].

Interventions: All subjects attended the physical therapy sessions three times per week for six weeks. Control group performed manual passive stretching exercise for hamstring and iliopsoas as well as back muscles. For hamstring and back muscle stretching exercise, the patient sat in a long sitting position with knees fully extended and the feet together free or in plantar flexion. We asked the patient to bend forward from the hips to reach towards the feet, with the head in flexion. The physical therapist came behind the patient and push him/her forward to stabilize the patient's position and knees and hold the position for 30 seconds, then relaxed for 30 seconds [29], with three repetitions per set, three sets per visit, and three visits per week for a period of six weeks.

For iliopsoas muscle stretching exercise, the patient lay in a crook lying position with both lower limbs hanging off the end of the table, holding one knee to the chest; the therapist stood in front of both lower limbs. One hand held the flexed knee, while the other hand stretched the other leg by pushing down and holding the position for 30 seconds, then relaxing for 30 seconds, with three repetitions per set, three sets per visit, and three visits per week for a period of six weeks.

An experimental group performed the same stretching exercise program as the control group, in addition to stretching exercise for the gastrocnemius muscle. For this exercise, the patient sat in a long sitting position, with knees fully extended and the ankles together in dorsiflexion, and the feet flat against the wall. We asked the pa- 
tient to bend forward from the hips to reach towards the feet with the head in flexion. The physical therapist then came behind the patient and pushed him/her forward to stabilize the patient's position and knees, holding the position for 30 seconds, then relaxing for 30 seconds, with three repetitions per set, three sets per visit, and three visits per week for a period of six weeks.

Strengthening exercises were performed in both groups. Sitting up from a supine position with hips and knees flexed was used for abdominal muscles strengthening exercises. Head and shoulders were lifted with a gradual curl to touch the knees with the hands. The abdominal muscle becomes more efficient. This isotonic abdominal exercise should begin with the hips and knees flexed. Strengthening exercises program for abdominal muscles were performed in sets of ten repetitions, ten seconds holding and ten to 15 seconds relaxing, ten sets per visit and three visits per week for a period of six weeks.

Stretching and strengthening exercises were undertaken for duration of an hour per session, three sessions per week for a period of 6 weeks. Participants in both groups received health education for low back pain by therapists, including correct posture and appropriate behavior changes to enhance functional outcomes as well as activities of daily living.

\section{Statistical Analysis}

Statistical analyses were performed after collecting the data and were treated statistically by using SPSS software (Statistical Package for the Social Sciences, version 18.0, SPSS Inc. Chicago, IL, USA). The following values were found: mean, standard deviation, $t$ value and $p$ value. An unpaired $t$-test was used for comparison between groups. The $p<0.05$ was considered significant.

\section{Results}

In this study, forty subjects participated with ages ranging from 25 to 40 years, and all of the subjects completed the study period. Table 1 shows patient demographics in both groups. There were no significant differences in patient demographics. Table 2 represents patient's clinical characteristics at baseline in both groups. Both groups were homogenous, and there were no significant differences regarding pain $(p=0.4449)$, Modified FFT $(p=0.5952)$, ODI $(p=0.2498)$, and lumbosacral angle measurements $(p=0.8336)$. Table 3 shows patient's post intervention clinical characteristics. There were significant differences between experimental and control groups at the end of the week six in all outcome measures. In the experimental group, the reduction was as follows: in terms of pain, from $7.65 \pm 1.225$ points to $0.35 \pm 0.489$ points $(p<0.001)$, modified (FTF) from $43.5 \pm$ 7.634 to $19.4 \pm 2.113$ ( $p<0.001)$, ODI from $80.25 \pm 9.808$ points to $6.9 \pm 5.784$ points $(p<0.001)$, and lumbosacral angle from $27.1 \pm 2.673$ to $21.1 \pm 2.292(p<0.001)$. In the control group, the reduction was as follows: in terms of pain, from $7.95 \pm 1.23$ points to $2.85 \pm 0.875$ points $(p<0.001)$, modified (FTF) from $44.9 \pm 7.622$ to $30.2 \pm 5.773(p<0.001)$, ODI from $76.45 \pm 10.956$ points to $50.35 \pm 11.249$ points $(p<0.001)$ and lumbosacral

Table 1. Patient's baseline demographics characteristics.

\begin{tabular}{cccc}
\hline Participants demographics & Control Group & Experimental Group & $p$-value \\
\hline Age (years) & $35.15 \pm 7.61$ & $34.45 \pm 7.45$ & 0.771 \\
Weight (kg) & $81.15 \pm 14.56$ & $76.5 \pm 16.92$ & 0.358 \\
Height (cm) & $169.45 \pm 7.76$ & $167.9 \pm 9.64$ & 0.579 \\
BMI (kg/cm $\mathbf{2})$ & $28.33 \pm 5.23$ & $27.22 \pm 6.05$ & 0.539 \\
\hline
\end{tabular}

Table 2. Visual Analogue Scale (VAS), Modified fingertip-to-floor test (Modified FFT), Oswestry disability index (ODI), Lumbosacral angle, Pre 1 (baseline for control group) and Pre 2 (baseline for experimental group).

\begin{tabular}{cccc}
\hline & Pre 1 & Pre 2 & \multicolumn{1}{c}{-value } \\
VAS & $7.95 \pm 1.23$ & $7.65 \pm 1.23$ \\
Modified FFT & $44.9 \pm 7.62$ & $43.5 \pm 7.63$ \\
ODI & $76.45 \pm 10.97$ & $80.25 \pm 9.81$ & 0.595 \\
Lumbosacral angle & $27.15 \pm 3.10$ & $27.10 \pm 2.67$ & 0.834 \\
\hline
\end{tabular}


Table 3. Visual Analogue Scale (VAS), Modified fingertip-to-floor test (Modified FFT), Oswestry disability index (ODI), Lumbosacral angle, Post 1 (post intervention for control group) and Post 2 (post intervention for experimental group).

\begin{tabular}{cccc}
\hline & Post 1 & Post 2 & p-value \\
VAS & $2.85 \pm 0.875$ & $0.35 \pm 0.489$ & 0.0001 \\
Modified FFT & $30.2 \pm 5.773$ & $19.40 \pm 2.113$ & 6.0001 \\
ODI & $50.35 \pm 11.249$ & $6.90 \pm 5.784$ & $21.10 \pm 2.292$ \\
Lumbosacral angle & $24.60 \pm 2.664$ & 0.0001 \\
\hline
\end{tabular}

angle from $27.15 \pm 3.099$ to $24.6 \pm 2.664(p<0.001)$.

\section{Discussion}

The results of this study showed significant differences between the control group and the experimental group in terms of pain, ROM of trunk flexion (Modified FFT), and Oswestry disability index (ODI). These differences can be explained by the assumption that the gastrocnemius shares in knee flexion, and its shortening will affect the length of hamstring and back muscles so, limiting forward bending and causing low back pain. Stretching of gastrocnemius leads to lengthening of this muscle that indirectly increases the length of the hamstrings and back muscle in the experimental more than control group. Thus, this lengthening increases ROM of forward trunk flexion and decreasing the pain.

The goals of treating chronic low back pain focus on improving the mechanical defects including shortening of the muscles and hyperlordosis, and this leads to reducing pain and improving functions. Patients often have unrealistic expectations of complete pain relief and full return to their previous level of activity. There is often a large gap between a patient's desired amount of pain reduction and the minimum percentage of improvement that would make a treatment worthwhile [30].

Many studies have concluded that imbalance of muscles causes the biomechanical defect of hyperlordotic posture in the lumbosacral region [31], kyphosis deformity in the sagittal plane of the sacrum [32], and incoordination of the lumbar-pelvic rhythm [33]. All of these problems lead to low back pain with restriction of ADL as restriction of forward bending, which combined with lumbar and hip motion, is considered one of the most important detriments resulting from limitation of lumbar-pelvic movement and shortening of hamstring muscles, due to their attachments to the posterior leg and to the ischial tuberosity [19] [20]. Forward bending is a coupled movement, combining lumbar flexion and pelvic rotation, called lumbarpelvic rhythm [13]. It results from coordinated activity between the back extensor muscles (erector spinae) and the hip extensor muscles (gluteus and hamstrings). Hamstring tightness is a common finding in LBP patients [34], and it has been argued that lengthening the hamstrings may allow greater motion to occur at the hips and therefore may reduce stress on the lumbar spine [35].

Subjects with shortened gastrocnemius muscles who perform the standard sit and reach test (SRT) with a $90^{\circ}$ ankle dorsiflexion can lead the physical therapist to attribute the subject's low score to shortened hamstring muscles. When the gastrocnemius is shortened, the subject feels discomfort in the gastrocnemius muscle before the hamstring muscle because thetension, created in the calf, is a limiting factor for continuing the SRTand preventing the subject from stretching the hamstring maximally due to great discomfort in the gastrocnemius. At this point, the extremities of the subject's gastrocnemius muscle are apart. Based on the anatomic position and function of the abdominal muscles, it has been speculated that abdominal muscle weakness produces an anterior pelvic tilt and lumbar hyperlordosis, resulting in LBP [36] [37]. In view of the above knowledge about the muscles stretch effects on SRT and also lumbopelvic rhythm leading to LBP, we must consider all these factors and causes to solve the pathomechnics of LBP.

Mechanical low back is considered a common problem and one of the causes of pain and restriction of daily activities. A physical therapy program in the form of a flexion program with stretching of back, hamstrings, and gastrocnemius in the same exercise, along with isolated stretching of iliopsoas muscles, was more beneficial in treatment, and we recommend considering stretching of gastrocnemius in the management of patients with chronic low back pain. This study has several limitations. A small sample size and short-term follow up limited our finings. Also, there was a difference in weight mean at baseline $(4.65 \mathrm{~kg})$ between both groups. Even it was not significant, it might affect the results. Further studies should be done on a larger sample with different types 
of stretching techniques, and should consider the long-term effect of stretching the gastrocnemius muscle on pain and function in patients with chronic low back pain. Furthermore, gastrocnemius-stretching exercises are varied and it may be more beneficial than others. An additional consideration is that some patients do not read and write English language, a panel of institutional expertshastranslated and validated Oswestery Disability Index into Arabic language, but has not been validated yet in a separate study. Consequently, any inaccuracies may affect the validity of one of our outcome measures. Further research should be conducted concerning translating and validating ODI into Arabic.

\section{Conclusion}

This study showed that manual stretching exercises for back, hamstring and iliopsoas muscles and strengthening exercises for abdominal muscle and postural instructions for activities of daily living were beneficial in decreasing pain and improving functional activities in people with chronic low back pain. Adding gastrocnemius manual stretching to regular stretching exercise for hamstring and iliopsoas as well as back muscles with strengthening exercise for abdominal muscles is more beneficial in reducing pain and improving functional activities for patients with chronic low back pain.

\section{Conflict of Interest}

All authors have no conflict of interest to declare in this study.

\section{References}

[1] Rozenberg, S. (2008) Chronic Low Back Pain: Definition and Treatment. La Revue du Praticien, 58, $265-272$.

[2] Hides, J.A., Richardson, C.A. and Jull, G.A. (1996) Multifidus Muscle Recovery Is Not Automatic after Resolution of Acute, First-Episode Low Back Pain. Spine (Phila Pa 1976), 21, 2763-2769. http://dx.doi.org/10.1097/00007632-199612010-00011

[3] Richardson, C. (1999) Spinal Segmental Stability. In: Therapeutic Exercise for Spinal Segmental Stabilization Low Back Pain, Churchill Livingstone, Edinburgh, 16-26.

[4] Isida, K. (2000) About Lumbar Stabilization Function-Comparison between the Normal Subjects and Low Back Pain. The Hokkaido Journal of Physical Therapy, 19, 15-18.

[5] McKenzie, R. (1981) The Lumbar Spine. In: Mechanical Diagnosis and Therapy. Spinal Publications, New Zealand, Waikanae, 155-168.

[6] Huang, Q., et al. (2013) The Intervention Effects of Different Treatment for Chronic Low Back Pain as Assessed by the Cross-sectional Area of the Multifidus Muscle. Journal of Physical Therapy Science, 25, 811-813. http://dx.doi.org/10.1589/jpts.25.811

[7] Waddell, G. and Grant I, G.A. (2000) Surgical Treatment of Lumbar Disc Prolapse and Degenerative Lumbar Disc Disease. In: Nachemson, J.E.A., Ed., Neck and Back Pain: The Scientific Evidence of Causes, Diagnosis, and Treatment, Williams and Wilkins, Philadelphia: Lippincott, 305-325.

[8] Bogduk, N. (2004) Management of Chronic Low Back Pain. The Medical Journal of Australia, 180, 79-83.

[9] Kendall, F.P., Provance, P.G., et al. (1993) Muscles: Testing and Function. Lippincott Williams \& Wilkins, Baltimore.

[10] Kawano, M.M., et al. (2010) Influence of the Gastrocnemius Muscle on the Sit-and-Reach Test Assessed by Angular Kinematic Analysis. Brazilian Journal of Physical Therapy, 14, 10-15. http://dx.doi.org/10.1590/S1413-35552010000100003

[11] Schafer, R. (1987) Clinical Biomechanics, Musculoskeletal Actions and Reactions. 2nd Edition, Williams and Wilkins, Baltimore.

[12] Fisk, J.W., Baigent, M.L. and Hill, P.D. (1984) Scheuermann’s Disease. Clinical and Radiological Survey of 17 and 18 Year Olds. American Journal of Physical Medicine, 63, 18-30.

[13] Norris, C.M. and Matthews, M. (2006) Correlation between Hamstring Muscle Length and Pelvic Tilt Range during Forward Bending in Healthy Individuals: An Initial Evaluation. Journal of Bodywork and Movement Therapies, 10, 122-126. http://dx.doi.org/10.1016/j.jbmt.2005.06.001

[14] Wilson, A. (1994) Are You Sitting Comfortably?-A Self-Help Guide for Sufferers of Back Pain, Neck Strain, Headaches, RSI, and Other Associated Problems. Random House, London.

[15] Esola, M.A., McClure, P.W., Fitzgerald, G.K. and Siegler, S. (1996) Analysis of Lumbar Spine and Hip Motion during Forward Bending in Subjects with and without a History of Low Back Pain. Spine, 21, 71-78. 
http://dx.doi.org/10.1097/00007632-199601010-00017

[16] McClure, P.W., Esola, M., Schreier, R. and Siegler, S. (1997) Kinematic Analysis of Lumbar and Hip Motion While Rising from a Forward, Flexed Position in Patients with and without a History of Low Back Pain. Spine, 22, 552-558. http://dx.doi.org/10.1097/00007632-199703010-00019

[17] Porter, J.L. and Wilkinson, A. (1997) Lumbar-Hip Flexion Motion. A Comparative Study between Asymptomatic and Chronic Low Back Pain in 18- to 36-Year-Old Men. Spine, 22, 1508-1513; Discussion 1513-1514. http://dx.doi.org/10.1097/00007632-199707010-00017

[18] Wong, T.K. and Lee, R.Y. (2004) Effects of Low Back Pain on the Relationship between the Movements of the Lumbar Spine and Hip. Human Movement Science, 23, 21-34. http://dx.doi.org/10.1016/j.humov.2004.03.004

[19] Koutedakis, Y., Frischknecht, R. and Murthy, M. (1997) Knee Flexion to Extension Peak Torque Ratios and Low-Back Injuries in Highly Active Individuals. International Journal of Sports Medicine, 18, 290-295. http://dx.doi.org/10.1055/s-2007-972636

[20] Li, Y., McClure, P.W. and Pratt, N. (1996) The Effect of Hamstring Muscle Stretching on Standing Posture and on Lumbar and Hip Motions during Forward Bending. Physical Therapy, 76, 836-845; Discussion 845-849.

[21] Ide, J., Maeda, S., Yamaga, M., Morisawa, K. and Takagi, K. (2003) Shoulder-Strengthening Exercise with an Orthosis for Multidirectional Shoulder Instability: Quantitative Evaluation of Rotational Shoulder Strength before and after the Exercise Program. Journal of Shoulder and Elbow Surgery, 12, 342-345. http://dx.doi.org/10.1016/S1058-2746(02)86886-0

[22] Dodd, K.J., Taylor, N.F. and Damiano, D.L. (2002) A Systematic Review of the Effectiveness of Strength-Training Programs for People with Cerebral Palsy. Archives of Physical Medicine and Rehabilitation, 83, 1157-1164. http://dx.doi.org/10.1053/apmr.2002.34286

[23] Wewers, M.E. and Lowe, N.K. (1990) A Critical Review of Visual Analogue Scales in the Measurement of Clinical Phenomena. Research in Nursing \& Health, 13, 227-236. http://dx.doi.org/10.1002/nur.4770130405

[24] Von Korff, M., Deyo, R.A., Cherkin, D. and Barlow, W. (1993) Back Pain in Primary Care. Outcomes at 1 Year. Spine, 18, 855-862. http://dx.doi.org/10.1097/00007632-199306000-00008

[25] Gauvin, M.G., Riddle, D.L. and Rothstein, J.M. (1990) Reliability of Clinical Measurements of Forward Bending Using the Modified Fingertip-to-Floor Method. Physical Therapy, 70, 443-447.

[26] Delitto, A., Erhard, R.E. and Bowling, R.W. (1995) A Treatment-Based Classification Approach to Low Back Syndrome: Identifying and Staging Patients for Conservative Treatment. Physical Therapy, 75, 470-485; Discussion 485489.

[27] Longo, U.G., Loppini, M., Denaro, L., Maffulli, N. and Denaro, V. (2010) Rating Scales for Low Back Pain. British Medical Bulletin, 94, 81-144. http://dx.doi.org/10.1093/bmb/ldp052

[28] Barrey, C., Jund, J., Noseda, O. and Roussouly, P. (2007) Sagittal Balance of the Pelvis-Spine Complex and Lumbar Degenerative Diseases. A Comparative Study about 85 Cases. European Spine Journal, 16, 1459-1467. http://dx.doi.org/10.1007/s00586-006-0294-6

[29] Bandy, W.D., Irion, J.M. and Briggler, M. (1997) The Effect of Time and Frequency of Static Stretching on Flexibility of the Hamstring Muscles. Physical Therapy, 77, 1090-1096.

[30] Yelland, M.J. and Schluter, P.J. (2006) Defining Worthwhile and Desired Responses to Treatment of Chronic Low Back Pain. Pain Medicine, 7, 38-45. http://dx.doi.org/10.1111/j.1526-4637.2006.00087.x

[31] Bookhout, M.R. (1993) Evaluation and Conservative Management of Spondylolisthesis. Journal of Back and Musculoskeletal Rehabilitation, 3, 24-31.

[32] Antoniades, S.B., Hammerberg, K.W. and De Wald, R.L. (2000) Sagittal Plane Configuration of the Sacrum in Spondylolisthesis. Spine, 25, 1085-1091. http://dx.doi.org/10.1097/00007632-200005010-00008

[33] Granata, K.P. and Sanford, A.H. (2000) Lumbar-Pelvic Coordination Is Influenced by Lifting Task Parameters. Spine, 25, 1413-1418. http://dx.doi.org/10.1097/00007632-200006010-00014

[34] Nourbakhsh, M.R. and Arab, A.M. (2002) Relationship between Mechanical Factors and Incidence of Low Back Pain. Journal of Orthopaedic \& Sports Physical Therapy, 32, 447-460. http://dx.doi.org/10.2519/jospt.2002.32.9.447

[35] Cailliet, R. (1994) Low Back Pain Syndrome. F.A. Davis, Philadelphia.

[36] Cailliet, R. (1981) Low Back Pain Syndrome. 3rd Edition, F.A. Davis, Philadelphia.

[37] Kendall, F.P., McCreary, E.K. and Provance, P.G. (1993) Muscle Testing and Function. 4th Edition, Lippincott, Williams and Wilkins, Philadelphia. 